CERN-TH 6925

IEM-FT-74/93

\title{
Effective Operators and Extended Symmetry
}

\author{
J.-M. Frère ${ }^{\dagger}$, M. Tytgat ${ }^{b}$ \\ Service de Physique Théorique \\ Université Libre de Bruxelles, CP 225 \\ Boulevard du Triomphe, B-1050 Bruxelles, Belgium \\ J.M. Moreno \\ Inst. de Estructura de la Materia \\ Serrano 123, E-28006 Madrid, Spain \\ J. Orloff $\sharp$ \\ Theory Division, CERN \\ CH-1211 Geneva 23, Switzerland
}

In this note we expand on our previous study of the implications of LEP1 results for future colliders. We extend the effective operator-based analysis of De Rújula et al. to a larger symmetry group, and show at which cost their expectations can be relaxed. Of particular interest to experiment is a rephrasing of our previous results in terms of the Renard et al. parametrization for the gauge boson self-couplings (slightly extended to include $\left.\delta g_{\gamma}\right)$. We suggest the use of a $\left(\delta g_{\gamma}, \delta g_{Z}\right)$ plot to confront the expectations of various models.

CERN-TH.6925/93

$07 / 93$

\footnotetext{
$\dagger$ Maître de Recherche FNRS, Corresponding Fellow, CERN.

b Aspirant FNRS.

\# e-mail: orloff@dxcern.cern.ch
} 


\section{Outline}

The purpose of the present paper is to investigate to which extent the extra degrees of freedom - possibly associated with light particles - resulting from an extended symmetry may alleviate the constraints on future high energy experiments resulting from the high precision measurements of LEP1 at the $Z$ peak.

In a previous note [1], we outlined the main characteristics of a minimal extension of the type $S U(2) \times U(1) \times U(1)^{\prime}$, and reached the tentative conclusion that the presence of a light $Z^{\prime}$ boson, with anomalous, gauge-invariant, dimension-6 couplings could remain undetected at LEP1 while providing, at least in terms of statistics, significant contributions already at the moderately higher energies to be reached by LEP2. In a way, this constitutes a loophole in the argument of [2], to the extent that some of the implicit conditions of [2] are not met; we acknowledge however quite willingly that the construction of this counterexample is involved enough to underline the intrinsic power of the general arguments $^{1}$ developed in [2].

We return to our previous analysis, with the purpose of expliciting somewhat the construction and providing our experimentalist colleagues with the basic tools to confront our approach with their Monte-Carlo simulations. In particular, we study the impact of our model on $M_{W}$ and LEP1 data like $\left(e_{A}, e_{V}\right)$, which determines the extreme values allowed for our parameters $\lambda$, the ratio of the $U(1)^{\prime}$ to the standard $U(1)$ couplings, and $\varepsilon$, the strength of the hypothetical anomalous coupling of the $Z^{\prime}$. In addition to the angular distribution of the produced $\mathrm{W}$ pairs, which is not directly accessible to experiment, we establish the relation of the extended model to the now usual parametrization of the amplitudes in terms of the variables $\left(\delta g_{Z}, \delta \kappa_{Z}, \delta \kappa_{\gamma}\right)$ introduced by Renard et al. (see for example [3]), which we need, however, to extend slightly by allowing for $\delta g_{\gamma}$. This reparametrization is not entirely trivial, as the approach of Renard et al. assumes that the $Z$ and $\gamma$ are the only neutral vector bosons. The $Z^{\prime}$ is thus simulated at the cost of introducing momentum-dependent form factors, both for the $Z$ and the $\gamma$ anomalous couplings. Fortunately, at tree level (and we limit the study of the extensions to their treelevel effects) these form factors only depend upon $\sqrt{s}$. In addition to the formal expression of the Renard parameters in terms of our fundamental Lagrangian variables, we work out typical values, as allowed by the latest LEP1 results [4].

\footnotetext{
1 at least those based on tree level considerations
} 


\section{General Considerations}

Effective Lagrangians provide a systematic way of parametrizing new physics beyond the Standard Model (SM) [5]. In such an approach, any new physical input merely boils down to a set of numerical coefficients weighting higher-dimensional (and hence nonrenormalizable) operators, constructed in terms of the low-energy fields alone. If the approach is to be of any use, ways must be found to truncate the infinite list of such operators down to a small subset. An obvious guide is the dimensionality of the operators. If indeed the new physics is supposed to originate at some large scale $\Lambda$, this scale is expected to appear in the denominator of the coefficient of each operator.

In gauge theories, the notion of gauge invariance of the operator basis has been abundantly discussed in the literature. This requirement has important consequences on the classification of operators according to dimension.

Let us consider indeed a typical example :

$$
O_{W B}^{\text {naive }}=W_{\mu \nu}^{3} B^{\mu \nu}
$$

This operator is apparently of dimension 4, although non-renormalizable, since it corresponds to a non-minimal coupling of the $W$. Quite obviously, this operator also violates gauge invariance, and even the global $S U(2)$ invariance of the effective Lagrangian. It has been argued that the notion of gauge invariance is of little relevance, since any noninvariant operator can be written in the form of a gauge-invariant term, evaluated in some unitary gauge after symmetry breaking. An obvious example to realize this consists in rewriting $O_{W B}^{\text {naive }}$ as

$$
O_{W B}=\phi^{+} \sigma^{i} \phi W_{\mu \nu}^{i} B^{\mu \nu}
$$

This term is now obviously gauge-invariant, and coincides (up to a dimensional coefficient) with the previous expression after symmetry breaking. This step however involves a change in the dimension of the operator considered, and stresses that it should, in a fully renormalizable framework, be penalized by two powers of the high scale. While the requirement of gauge invariance of the effective Lagrangian (before symmetry breaking) is not a mathematical necessity, we thus observe that, on physical grounds, it is a useful guide in tracking down the severity of the actual dependence upon the high scale.

The enforcement of gauge invariance of the operator set, and the restriction to operators of dimension $\leq 6$, has allowed De Rújula et al. [2] to establish powerful constraints 
on the possible observations at future colliders. While we will return to these assumptions, we briefly outline our current understanding of the situation. The reason for these constraints is simple. Gauge invariance typically relates Feynman vertices with different numbers of gauge fields; for instance in the case of $O_{W B}$ above, terms with external legs $B W W$ and $B W$ are automatically related. Owing to its high luminosity at the $Z$-peak LEP1 has brought strong constraints on the masses and couplings of the gauge bosons - thus constraining severely the 2-point functions. As a result, operators leading to both 2 -gauge and 3 -gauge boson terms are severely constrained already at the tree level. However, "blind directions" also exist at tree level. In particular, such is trivially the case of operators which do not contain any $2-W$ contributions, and the archetypal example is:

$$
O_{W}=\frac{1}{3 !} \varepsilon_{i j k} W_{\mu}^{\nu j} W_{\nu}^{\lambda k} W_{\lambda}^{\mu i}
$$

In addition to the undisputable successes of the tree-level considerations, several groups have studied further constraints arising from the inclusion of the new operators in 1-loop diagrams[6], typically generating contributions to low-energy parameters measured to a high accuracy (for instance $(g-2)$, or the electric dipole moment of various leptons and quarks). These studies show that, in a number of cases, tree-level blind directions can bring important 1-loop contributions, and can therefore be partly excluded. While these considerations are highly plausible, it should of course be kept in mind that the same high-energy contributions, which are responsible for anomalous $\mathrm{W}$ operators, can also -and in general do- generate contact terms, providing direct contributions to these parameters. Such terms are furthermore often required to regulate the divergences due to the inclusion of effective higher-dimension operators in loops. The presence of such uncontrolled counterterms thus precludes in general firm conclusions based on loop contributions alone.

The situation may appear gloomy for some of the often advertised purposes of future accelerators. We want however to emphasize that any effective Lagrangian approach assumes in some way the existence of a "desert" between the exactly treated "known" lowenergy scale, and the high-energy scale, described only by effective operators. That the harvest of new interactions in such a desert be meagre, should then not be too surprising.

Reducing the scale at which new physics sets in can be treated in two ways. The most obvious one is to to include higher-dimensional operators in the analysis; this however quickly makes the model completely unpredictable, since there is a large number of operators beyond dimension 6 . The other way to see things is to insist right away on the 
presence of further particles in the basic spectrum (think for instance of a possible $Z^{\prime}$ ). Here also, the previous approach has to be reviewed. Even if only dimension-6 operators are constructed, these must involve new fields and, correspondingly, more degrees of freedom are injected. Actually, this may be seen as a curious twist on one of the basic assumptions usually made. It is customary indeed to assume only the minimal $S U(2) \times U(1)$ symmetry to classify the additional effective operators. At first sight, this seems to be a liberal assumption, since any larger symmetry is expected to introduce more constraints on the form of the Lagrangian. The argument fails, however, if the extra symmetry considered, as is often the case, imposes the presence of an enriched intermediate-energy particle spectrum, allowing us to write down a much larger set of operators.

We are interested in building such an example, just to check how much our expectations for physics at new accelerators would be affected. We present here in some detail the simplest case we could think of, namely the extension of the original symmetry group to $S U(2) \times U(1) \times U(1)^{\prime}$, with a relatively light $Z^{\prime}$. We note in passing that the first $U(1)$ being unchanged, the gauge boson associated with $U(1)^{\prime}$ is orthogonal to the photon, which reduces from the onset any possible consequences on low energy anomalous magnetic or eletric dipole moments.

Our purpose in this note is to go back to the fundamental assumptions, and to examine from the start whether any escape from the above-sketched analysis is indeed possible.

\section{The Model}

We thus proceed in two steps, as in [1]. First, we add the extra Abelian gauge boson $B^{\prime}$, together with a scalar $\chi$ providing for its mass. Right-handed neutrinos $\left(\nu_{R}\right)$ also need to be introduced, which, except for the cancellation of anomalies, play no role in our considerations. The matter content and the additional hypercharges are summarized in Table 1. Two more parameters are needed to determine the modified neutral current Lagrangian: the strength of the new interaction $\left(g_{1}^{\prime}\right)$ and the mass of the new gauge boson $\left(M_{Z^{\prime}}\right)$. In this minimal model, we have fixed the scalar content and as a result the extra mixing angle $\left(\theta_{3}\right)$ between $Z$ and $Z^{\prime}$ is not an independent parameter. For the couplings, it is convenient to turn to the variables $e, \theta_{W}$ and $\lambda\left(e=g_{2} s_{W}=g_{1} c_{W}, \lambda=s_{W} g_{1}^{\prime} / g_{1}\right)$.

But this extra symmetry might not be the end of physics, and we next parametrize our further ignorance by an effective operator approach. A typical new dimension- 6 operator is obtained by replacing the $B$ of $O_{W B}$ by a $B^{\prime}$ field, yielding

$$
\mathcal{L}_{W B^{\prime}} \doteq \frac{\varepsilon}{v^{2}} O_{W B^{\prime}}=\frac{\varepsilon}{v^{2}} \phi^{+} \sigma^{i} \phi W_{\mu \nu}^{i} B^{\prime \mu \nu} .
$$


With the introduction of the new operator $O_{W B^{\prime}}$ and after S.B., the bilinear part of the Lagrangian for the neutral gauge bosons becomes:

$$
\mathcal{L}_{N G B}=-\frac{1}{4} W_{\mu \nu} \mathcal{K} W^{\mu \nu}+\frac{1}{2} W_{\mu} \mathcal{M}^{2} W^{\mu}
$$

where

$$
\begin{aligned}
& W_{\mu \nu}=\left(\begin{array}{lll}
\hat{W}_{\mu \nu}^{3} & B_{\mu \nu} & B_{\mu \nu}^{\prime}
\end{array}\right) \\
& \hat{W}_{\mu \nu}^{3}=\partial_{\mu} W_{\nu}^{3}-\partial_{\nu} W_{\mu}^{3}
\end{aligned}
$$

with $^{2}$

$$
\mathcal{K}=\left(\begin{array}{lll}
1 & 0 & \varepsilon \\
0 & 1 & 0 \\
\varepsilon & 0 & 1
\end{array}\right)
$$

and

$$
\mathcal{M}^{2}=\left(\begin{array}{ccc}
\frac{1}{4} g_{2}^{2} v^{2} & -\frac{1}{4} g_{2} g_{1} v^{2} & \frac{1}{5} g_{2} g_{1}^{\prime} v^{2} \\
-\frac{1}{4} g_{2} g_{1} v^{2} & \frac{1}{4} g_{1}^{2} v^{2} & -\frac{1}{5} g_{1} g_{1}^{\prime} v^{2} \\
\frac{1}{5} g_{2} g_{1}^{\prime} v^{2} & -\frac{1}{5} g_{1} g_{1}^{\prime} v^{2} & \frac{4}{25} g_{1}^{\prime 2} v^{2}+g_{1}^{\prime 2} V^{2}
\end{array}\right)
$$

We need to diagonalize simultaneously the mass and the kinetic matrices for neutral fields, ending up with the canonical form for the latter. In addition to rotations in $W_{\mu-}$ space, this involves a rescaling of the fields. We thus define $\mathcal{S}$ relating the physical neutral fields to the original gauge fields ${ }^{3}$ by:

$$
\begin{aligned}
\mathcal{S}^{t} \mathcal{K} \mathcal{S} & =\mathbf{1} \\
\mathcal{S}^{t} \mathcal{M}^{2} \mathcal{S} & =\mathcal{M}_{d}^{2} \\
\left(\begin{array}{c}
W_{\mu}^{3} \\
B_{\mu} \\
B_{\mu}^{\prime}
\end{array}\right) & =\mathcal{S} \cdot\left(\begin{array}{c}
A_{\mu} \\
Z_{\mu} \\
Z_{\mu}^{\prime}
\end{array}\right)
\end{aligned}
$$

To get a feeling for what we can expect as a function of the various parameters, let us explore $\mathcal{S}$ in the limit of small $\varepsilon$. To first order in $\varepsilon$, we can write:

$$
\begin{aligned}
& \mathcal{K}=\mathbf{1}+\varepsilon \Delta \\
& \mathcal{S}=\mathcal{O}_{W}^{t} \mathcal{O}_{3}^{t}(\mathbf{1}+\varepsilon \mathcal{G})
\end{aligned}
$$

2 Notice that the use of $v^{2}$ (instead of a higher energy scale $\Lambda^{2}$ ) as a dimensionalizer in (3.1) reflects our phenomenological, low-energy approach. With this choice, an $|\varepsilon| \ll \varepsilon_{\text {crit }}=1$ has small effects, $\varepsilon_{\text {crit }}$ being the extreme value that makes $\mathcal{K}$ degenerate. We do not wish to address the question of the origin of this $\varepsilon$ here.

3 This redefinition matrix $\mathcal{S}$ is not unitary. 
where

$$
\mathcal{O}_{W}=\left(\begin{array}{ccc}
s_{W} & c_{W} & 0 \\
-c_{W} & s_{W} & 0 \\
0 & 0 & 1
\end{array}\right)
$$

and

$$
\mathcal{O}_{3}=\left(\begin{array}{ccc}
1 & 0 & 0 \\
0 & c_{3} & -s_{3} \\
0 & s_{3} & c_{3}
\end{array}\right)
$$

is the standard rotation matrix that describes the $Z-Z^{\prime}$ mixing for $\varepsilon=0$. Then, from (3.6) we get two equations for $\mathcal{G}$ :

$$
\begin{gathered}
\mathcal{G}+\mathcal{G}^{t}+\Delta^{\prime}=0 \\
\left.\mathcal{M}_{d}^{2}\right|_{\varepsilon=0} \mathcal{G}+\left.\mathcal{G}^{t} \mathcal{M}_{d}^{2}\right|_{\varepsilon=0}=\mathcal{M}_{d}^{2}-\left.\mathcal{M}_{d}^{2}\right|_{\varepsilon=0}
\end{gathered}
$$

with

$$
\Delta^{\prime}=\mathcal{O}_{3} \mathcal{O}_{W} \Delta \mathcal{O}_{W}^{t} \mathcal{O}_{3}^{t}=\left(\begin{array}{ccc}
0 & -s_{3} s_{W} & c_{3} s_{W} \\
-s_{3} s_{W} & 2 c_{3} s_{3} c_{W} & -\left(c_{3}^{2}-s_{3}^{2}\right) c_{W} \\
c_{3} s_{W} & -\left(c_{3}^{2}-s_{3}^{2}\right) c_{W} & -2 c_{3} s_{3} c_{W}
\end{array}\right)
$$

The solution is:

$$
\mathcal{G}=\left(\begin{array}{ccc}
0 & s_{3} s_{W} & -c_{3} s_{W} \\
0 & -c_{3} s_{3} c_{W} & l\left(c_{3}^{2}-s_{3}^{2}\right) c_{W} \\
0 & -(l-1)\left(c_{3}^{2}-s_{3}^{2}\right) c_{W} & c_{3} s_{3} c_{W}
\end{array}\right)
$$

with $l=M_{Z^{\prime}}^{2} /\left(M_{Z^{\prime}}^{2}-M_{Z}^{2}\right)$.

The diagonalization of $\mathcal{M}^{2}$ yields the following masses for $Z$ and $Z^{\prime}$ :

$$
\begin{gathered}
M_{Z}^{2}=\left.M_{Z}^{2}\right|_{\varepsilon=0}\left(1-2 c_{3} s_{3} c_{W} \varepsilon\right) \\
M_{Z^{\prime}}^{2}=\left.M_{Z^{\prime}}^{2}\right|_{\varepsilon=0}\left(1+2 c_{3} s_{3} c_{W} \varepsilon\right)
\end{gathered}
$$

Notice that if $\theta_{3}=0$, then $\Delta$ has zeros in the diagonal and from (3.12), $\mathcal{G}$ has the same property. Then, from (3.10)

$$
\delta\left(M_{i}^{2}\right)=\Sigma_{j} \mathcal{G}_{i j} M_{j}^{2} \delta_{j i}=0
$$

and the masses are not corrected. ${ }^{4}$

\footnotetext{
4 This is a general result: when the canonical kinetic term is changed without modifying the diagonal terms in the base which diagonalizes the gauge boson mass matrix, these masses remain unchanged at first order and only a redefinition of the fields is induced.
} 
The lesson is that the impact of $\varepsilon$ on the eigenvalues is only of order $\theta_{3} \varepsilon$ in the limit of small $\varepsilon$. In particular, the influence of $\varepsilon$ on the observable $M_{Z}$ may be masked by a small value of $\theta_{3}$.

The value of $\theta_{W}$ is obtained by solving:

$$
\begin{aligned}
\left.\left(M_{Z}^{2}+M_{Z^{\prime}}^{2}\right)\right|_{\varepsilon=0} & =\frac{4 \pi \alpha}{s_{W}^{2} c_{W}^{2}}\left[v^{2}\left(\frac{1}{4}+\frac{4}{25} \lambda^{2}\right)+\lambda^{2} V^{2}\right] \\
\left.M_{Z}^{2} M_{Z^{\prime}}^{2}\right|_{\varepsilon=0} & =\frac{\pi \alpha}{s_{W}^{2} c_{W}^{2}} \lambda^{2} v^{2} V^{2}
\end{aligned}
$$

for $v^{2}$. Taking into account that

$$
v^{2}=\frac{1}{\sqrt{2} G_{\mu}} \frac{1}{1-\Delta r}
$$

$\theta_{W}$ can be extracted as a function of the fundamental parameters $\alpha, G_{\mu}, M_{Z}, M_{Z^{\prime}}, \lambda, \varepsilon$ and of $\theta_{3}$ (although this one is not independent in our model) and obeys:

$$
\sin \theta_{3}=\frac{4 \lambda D / 5}{\left.\sqrt{\left(M_{Z^{\prime}}^{2}-D\right)\left(M_{Z^{\prime}}^{2}-M_{Z}^{2}\right)}\right|_{\varepsilon=0}}
$$

where

$$
D=\frac{\pi \alpha}{s_{W}^{2} c_{W}^{2}} \frac{1}{\sqrt{2} G_{\mu}} \frac{1}{1-\Delta r} .
$$

Again, the variation of $\sin ^{2} \theta_{W}$ only probes the $\varepsilon \theta_{3}$ combination:

$$
\sin ^{2} \theta_{W}=\left.\sin ^{2} \theta_{W}\right|_{\varepsilon=0}\left(1-2 \varepsilon c_{3} s_{3} \frac{c_{W}^{3}}{c_{W}^{2}-s_{W}^{2}}\right)
$$

We have given above the corrections induced by a small $\varepsilon$. Another interesting limit is to take $\lambda$ small relative to $\varepsilon$ and to solve for the $\mathcal{S}$ matrix exactly in $\varepsilon$. The effects on the low-energy observables would then be of order $\varepsilon \tilde{\theta}_{3}$ where $\tilde{\theta}_{3}$ is a mixing angle of $O\left(\varepsilon M_{Z}^{2} / M_{Z}^{\prime 2}\right)$.

If $\lambda$ and $\varepsilon$ are of the same order and the $Z^{\prime}$ is not very massive, the situation is more complicated and the matrix $S$ must be evaluated numerically. If it is massive enough, a heavy $Z^{\prime}$ decouples, as both $\theta_{3}$ and $\tilde{\theta}_{3}$ become negligible, and the matrix $\mathcal{S}$ reduces to the very simple form

$$
\mathcal{S}=\left(\begin{array}{ccc}
s_{W} & -c_{W} & -\varepsilon \\
c_{W} & s_{W} & 0 \\
0 & 0 & 1
\end{array}\right)
$$

which will be useful in the following. 
To study the process $e^{+} e^{-} \rightarrow W^{+} W^{-}$we finally need the Feynman rules for neutral currents and Three-Gauge-boson-Vertices (TGV). Those are deduced from

$$
\mathcal{L}_{N C}=\left(\begin{array}{lll}
J_{\mu}^{3} & J_{\mu}^{Y} & J_{\mu}^{Y^{\prime}}
\end{array}\right) \cdot \operatorname{diag}\left(\begin{array}{lll}
g_{2} & g_{1} & g_{1^{\prime}}
\end{array}\right) \cdot\left(\begin{array}{c}
W_{3}^{\mu} \\
B^{\mu} \\
B^{\prime \mu}
\end{array}\right)
$$

and

$$
\mathcal{L}_{T G V}=-i g_{V}\left[\left(\hat{W}_{\mu \nu}^{+} W^{\mu-}-\hat{W}_{\mu \nu}^{-} W^{\mu+}\right) V^{\nu}+\kappa_{V} W_{\mu}^{+} W_{\nu}^{-} V^{\mu \nu}\right]
$$

where $V=\gamma, Z, Z^{\prime}$, and $\hat{W}_{\mu \nu}$ is defined from (3.3).

From the definition of $\mathcal{S}$ and $O_{W B^{\prime}}$ the couplings of the physical gauge bosons are

$$
\begin{aligned}
\left(g^{\gamma}, g^{Z}, g^{Z^{\prime}}\right) & =\left(g_{2}, 0,0\right) \cdot \mathcal{S} \\
\left(g^{\gamma} \kappa^{\gamma}, g^{Z} \kappa^{Z}, g^{Z^{\prime}} \kappa^{Z^{\prime}}\right) & =\left(g_{2}, 0, g_{2} \varepsilon\right) \cdot \mathcal{S} \\
\left(e_{L(R)}^{\gamma}, e_{L(R)}^{Z}, e_{L(R)}^{Z^{\prime}}\right) & =\left(g_{2} T_{e_{L(R)}}^{3}, g_{1} Y_{e_{L(R)}}^{B}, g_{1}^{\prime} Y_{e_{L(R)}}^{B^{\prime}}\right) \cdot \mathcal{S} \\
e_{V(A)} & =\frac{1}{2}\left(e_{R}+(-) e_{L}\right),
\end{aligned}
$$

following ${ }^{5}$ the notation of [1].

The expression of the differential cross-section for the process $e^{+} e^{-} \rightarrow W^{+} W^{-}$can be found in [7] and its generalization to the enlarged gauge group $S U(2) \times U(1) \times U(1)^{\prime}$ as a function of the previous parameters given in [1] is repeated here for the sake of completeness:

$$
\frac{d \sigma\left(e^{+} e^{-} \rightarrow W^{+} W^{-}\right)}{d \cos \theta}=s^{1 / 2}\left(s / 4-M_{W}^{2}\right)^{1 / 2}|A|^{2},
$$

where $A$ takes into account the contributions from the four usual diagrams corresponding to the $t$-channel $\nu$ exchange and the $s$-channel $\gamma, Z, Z^{\prime}$ exchanges:

$$
|A|^{2}=\frac{1}{8 \pi} \sum_{\alpha, \beta}\left(a_{\alpha}^{V} \operatorname{Spin}_{\alpha, \beta} a_{\beta}^{V}+a_{\alpha}^{A} \operatorname{Spin}_{\alpha, \beta} a_{\beta}^{A}\right)
$$

with

$$
a_{\alpha=\nu, \gamma, Z, Z^{\prime}}^{V(A)}=\left(\frac{g_{2}^{2}}{4 t}, \frac{e_{V(A)}^{\gamma} g^{\gamma}}{s}, \frac{e_{V(A)}^{Z} g^{Z}}{s-M_{Z}^{2}}, \frac{e_{V(A)}^{Z^{\prime}} g^{Z^{\prime}}}{s-M_{Z^{\prime}}^{2}}\right)
$$

\footnotetext{
5 up to the correction of a trivial misprint
} 
and

$$
\begin{aligned}
\operatorname{Spin}_{\nu, \nu}= & {\left[\frac{u t}{M_{W}^{4}}-1\right]\left[\frac{t^{2}}{4 s^{2}}+\frac{M_{W}^{4}}{s^{2}}\right]+\frac{t^{2}}{s M_{W}^{2}} } \\
\operatorname{Spin}_{\nu, i}= & {\left[\frac{u t}{M_{W}^{4}}-1\right]\left[\frac{\kappa_{i} t}{4 s}-\frac{M_{W}^{2} t}{2 s^{2}}-\frac{M_{W}^{4}}{s^{2}}\right]+\left(1+\kappa_{i}\right)\left[\frac{t}{2 M_{W}^{2}}-\frac{t}{s}+\frac{M_{W}^{2}}{s}\right] } \\
\operatorname{Spin}_{i, j}= & {\left[\frac{u t}{M_{W}^{4}}-1\right]\left[\frac{\kappa_{i} \kappa_{j}}{4}-\frac{M_{W}^{2}}{s} \frac{\left(1+\kappa_{i} \kappa_{j}\right)}{2}+3 \frac{M_{W}^{4}}{s^{2}}\right] } \\
& +\left(1+\kappa_{i}\right)\left(1+\kappa_{j}\right)\left[\frac{s}{4 M_{W}^{2}}-1\right] ; \quad i=\gamma, Z, Z^{\prime}
\end{aligned}
$$

describing the angular dependence.

\section{Calculation of the Parameters "à la Renard"}

The observability of the differential cross-section (3.25) was however only discussed [1] in terms of raw statistics, without considering, for example, the explicit angular resolution of the detectors. As future experiments are often modelled directly in terms of the final observable particles (leptons, missing momenta or jets) and related to fundamental Lagrangians in terms of the Renard et al. parameters, e.g. $\left(\delta g_{Z}, \delta \kappa_{Z}, \delta \kappa_{\gamma}\right)$, we found it useful to provide the relation between the present approach and this common formulation. The apparent difficulty here stems from the fact that the Renard parametrization was written in full generality only for the case of two neutral gauge bosons ( $Z$ and $\gamma$ ), while we need here to take into account the $Z^{\prime}$.

As we show below, it is fortunately possible (for this particular channel) to reabsorb the $Z^{\prime}$ exchange amplitude into a redefinition of the $\gamma$ - and $Z$-couplings to the $W$ 's; the price to pay is that the deviations from the standard model, $\delta g^{Z}, \delta \kappa^{Z}$ and $\delta \kappa^{\gamma}$ now become $s$-dependent. Moreover, since $e_{L}^{Z^{\prime}}$ and $e_{R}^{Z^{\prime}}$ differ from their $Z$ counterparts, we will need to introduce a non-zero $\delta g^{\gamma}$ as well. Notice that this effective parameter is only a convenient effective way to mimic the presence of the $Z^{\prime}$, and in no way implies a modification of the $W$ 's electric charge. We think however that the necessity to introduce this extra parameter in the neutral couplings of $W$ 's is interesting in itself and may be more general than the present framework. We therefore urge experimentalists to take this extra freedom into account in further studies.

We now proceed as announced to integrate out the propagator of the $Z^{\prime}$ into a redefinition of the $Z$ and $\gamma$ couplings to the $W^{\prime}$ 's. We temporarily affect these new couplings 
with a tilde; writing down the different contributions to the cross-section, we impose the conditions

$$
\begin{gathered}
\sum_{j=\gamma, Z} \frac{e_{L, R(S M)}^{j} \tilde{g}^{j}}{s-M_{j}^{2}}=\sum_{i=\gamma, Z, Z^{\prime}} \frac{e_{L, R}^{i} g^{i}}{s-M_{i}^{2}} \\
\sum_{j=\gamma, Z} \frac{e_{L, R(S M)}^{j} \tilde{g}^{j} \tilde{\kappa}^{j}}{s-M_{j}^{2}}=\sum_{i=\gamma, Z, Z^{\prime}} \frac{e_{L, R}^{i} g^{i} \kappa^{i}}{s-M_{i}^{2}}
\end{gathered}
$$

where

$$
\tilde{\kappa}^{j}=\kappa^{j} ; \tilde{g}^{j}=g^{j}
$$

in the decoupling limit $M_{Z^{\prime}} \rightarrow \infty$.

In addition to an explicit pole, the presence of the $Z^{\prime}$ also affects the mass and the couplings of the $Z$ by terms of order $\theta_{3}$, as we have already seen. While we take these effects fully into account to determine the compatibility of the present model with LEP1 data, and for all the plots reproduced below, we find it convenient to neglect $\delta e_{L, R}^{Z}=e_{L, R}^{Z}-e_{L, R(S M)}^{Z}$ (which is amply justified by the smallness of the deviations, see the plots of $\delta e_{A, V}$ below) to provide more readable analytic results. This allows us to rewrite the equation (4.1) in the following simple form

$$
\begin{gathered}
\sum_{j=\gamma, Z} \frac{e_{L, R}^{j}\left(\tilde{g}^{j}-g^{j}\right)}{s-M_{j}^{2}}=\frac{e_{L, R}^{Z^{\prime}} g^{Z^{\prime}}}{s-M_{Z^{\prime}}^{2}} \\
\sum_{j=\gamma, Z} \frac{e_{L, R}^{j}\left(\tilde{g}^{j} \tilde{\kappa}^{j}-g^{j} \kappa^{j}\right)}{s-M_{j}^{2}}=\frac{e_{L, R}^{Z^{\prime}} g^{Z^{\prime}} \kappa^{Z^{\prime}}}{s-M_{Z^{\prime}}^{2}}
\end{gathered}
$$

In the same approximation as above, and now introducing the convenient notation $\delta g$ instead of $\tilde{g}$, we have:

$$
\delta g^{j} \doteq \tilde{g}^{j}-g_{(S M)}^{j} \approx \tilde{g}^{j}-g^{j}+O\left(\varepsilon \theta_{3}, \varepsilon \tilde{\theta}_{3}\right)
$$

(and similarly for $g^{j} \kappa^{j}$ ), thus parametrizing the effects of the $Z^{\prime}$ as a departure from the SM's prediction. Solving what now are systems of two equations with two unknowns yields

$$
\begin{aligned}
\delta g^{Z} & =g^{Z^{\prime}} \cdot \frac{e_{L}^{Z^{\prime}}-e_{R}^{Z^{\prime}}}{e_{L}^{Z}-e_{R}^{Z}} \cdot \frac{s-M_{Z}^{2}}{s-M_{Z^{\prime}}^{2}} \\
\delta g^{\gamma} & =g^{Z^{\prime}} \cdot \frac{e_{L}^{Z} e_{R}^{Z^{\prime}}-e_{R}^{Z} e_{L}^{Z^{\prime}}}{e^{\gamma}\left(e_{L}^{Z}-e_{R}^{Z}\right)} \cdot \frac{s}{s-M_{Z^{\prime}}^{2}}
\end{aligned}
$$


and

$$
\begin{gathered}
\delta\left(g^{Z} \kappa^{Z}\right)=g^{Z^{\prime}} \kappa^{Z^{\prime}} \cdot \frac{e_{L}^{Z^{\prime}}-e_{R}^{Z^{\prime}}}{e_{L}^{Z}-e_{R}^{Z}} \cdot \frac{s-M_{Z}^{2}}{s-M_{Z^{\prime}}^{2}} \\
\delta\left(g^{\gamma} \kappa^{\gamma}\right)=g^{Z^{\prime}} \kappa^{Z^{\prime}} \cdot \frac{e_{L}^{Z} e_{R}^{Z^{\prime}}-e_{R}^{Z} e_{L}^{Z^{\prime}}}{e^{\gamma}\left(e_{L}^{Z}-e_{R}^{Z}\right)} \cdot \frac{s}{s-M_{Z^{\prime}}^{2}}
\end{gathered}
$$

From there on, solving for the $\mathcal{S}$ matrix in the appropriate limit and using (3.24) for the couplings yields the expected deviation from the SM for $W$-pair production ${ }^{6}$. For instance, the simplest analytical expression for $\mathcal{S}$, i.e. (3.21), in the limit of decoupling $Z^{\prime}$ $\left(M_{Z}^{\prime} \gg M_{Z}\right)$, gives:

$$
\begin{aligned}
\delta g^{Z} & =e \varepsilon\left(\frac{4 \lambda}{5 s_{W}}-\varepsilon \frac{c_{W}}{s_{W}}\right) \cdot \frac{s-M_{Z}^{2}}{s-M_{Z^{\prime}}^{2}} \\
\delta g^{\gamma} & =e \varepsilon\left(\frac{\lambda}{5 c_{W}^{2} s_{W}}\left(1+4 s_{W}^{2}\right)-\varepsilon \frac{s_{W}}{c_{W}}\right) \cdot \frac{s}{s-M_{Z^{\prime}}^{2}}
\end{aligned}
$$

and in this limiting situation

$$
\delta\left(g^{Z} \kappa^{Z}\right)=\delta\left(g^{\gamma} \kappa^{\gamma}\right)=0
$$

Though being rather imprecise for the parameter region we are ultimately interested in, (4.7) gives a good qualitative understanding of the effects of the $Z^{\prime}$ and the extra anomalous coupling. It is interesting to note that, in this limit, a non-vanishing $g^{Z^{\prime}}$ persists to order $\varepsilon$, while the $g^{Z^{\prime}} \kappa^{Z^{\prime}}$ vanishes. This is due to the gauge invariance of the operator $O_{W B^{\prime}}$. Had we used $\hat{W}_{\mu \nu}$ instead of $W_{\mu \nu}$ in (2.2), the fixed-angle, large- $s$ cross-section would have terms diverging like $s\left(\frac{\kappa^{Z^{\prime}}{ }_{s}}{M_{Z^{\prime}}{ }^{\prime}}\right)^{2}$. These cannot be compensated by the $t$-channel which, in the decoupling limit, has to cancel the similar terms from the $\gamma$ and $Z$ exchanges: another example of gauge invariance enforcing a sound high-energy behaviour [2]. This observation persists to some extent in the non-decoupling situation, as is apparent from the numerical results below: the $\delta(g \kappa)$ 's remain much smaller than the $\delta g$ 's.

\section{LEP1 Constraints and Observability}

A clean way to check for the allowed range of our extra parameters (namely $\lambda$ and $\varepsilon$, for any given value of $M_{Z^{\prime}}$ ) is obviously to proceed to a $\chi^{2}$ fit to the bulk of experimental data.

${ }^{6}$ One should note that this parametrization is not universal and is clearly not applicable as such, for example, to $W$-pair production at hadron colliders or muons pairs production. 
Since the working of such an analysis is unfortunately not very easy to grasp intuitively, we rather present a simpler exercise. Namely, we will study here the impact of $\lambda$ and $\varepsilon$ on the most sensitive parameters of the Standard Model, namely the vector and axial couplings $\left(e_{A}, e_{V}\right)$ of the leptons to the $Z$, as well as their reflection on the $W$ mass (we remind the reader that we take the standard option of fixing $M_{Z}, \alpha$ and the muon decay constant $G_{\mu}$ ). In the graphs presented below, we have (somewhat abusively) assumed that $\varepsilon=\lambda=0$ corresponds to the optimum Standard Model value, and compared the changes in $e_{A}, e_{V}$, $M_{W}$ to the current error bar [4]. The results are presented for $M_{Z^{\prime}}=210$ or $300 \mathrm{GeV}^{7}$ (see fig. 1). As seen from those graphs, even for as low a $Z^{\prime}$ mass as $210 \mathrm{GeV}, \varepsilon \in[-0.1,0.1]$ is allowed for $\lambda \leq 0.1$. We have checked that these conclusions are consistent with a more refined $\chi^{2}$ analysis.

Having established the LEP1-allowed range for our extra parameters, as a function of $M_{Z^{\prime}}$, we now proceed to plot the expected effects at higher-energy colliders, taking as a standard example LEP2 at $\sqrt{s}=200 \mathrm{GeV}$. A typical plot of the differential crosssection can be found in [1]. Note that according to the conventions of [7] we take $\theta$ to be the emission angle of the $W^{+}$with respect ot the incoming $e^{-}$. The integrated cross-section suffers from cancellations between the $\nu-Z^{\prime}$ and the $(\gamma, Z)-Z^{\prime}$ interference terms, as expected from gauge invariance. A decrease in the backward cross-section partly compensates an increase in the forward region, this compensation being exact around an angle of $135^{\circ}$, depending on the parameters. It is therefore useful, if we want to pursue the analysis in terms of cross-sections, to define an asymmetry, which crystallizes in one single number this peculiar angular dependence:

$$
A_{135} \doteq \frac{\sigma_{\theta>135}-\sigma_{\theta<135}}{\sigma_{\text {tot }}}
$$

Since $\theta=135^{\circ}$ also happens to roughly equal the median of the angular distribution at $200 \mathrm{GeV}$, this asymmetry is only penalized by a factor of $\sqrt{2}$ for its statistical errors with respect to the total cross-section, and yet it is most sensitive to the $Z^{\prime}$ and $\varepsilon$ effects. On the basis of $10^{4}$ events, this corresponds to an optimal (i.e. statistical) $1 \sigma$-sensitivity of $1.4 \%$ for this asymmetry, and there is thus room for $2 \sigma$ effects within the range of LEP1acceptable parameters, even for $M_{Z^{\prime}}$ as high as $300 \mathrm{GeV}$ (see [1]). For $M_{Z^{\prime}}=210 \mathrm{GeV}$,

7 Notice that such low values of $M_{Z}^{\prime}$ are not excluded, provided $\lambda$ is small enough: for most $Z^{\prime}$ models, $\lambda=O(1)$, and masses smaller than $400 \mathrm{GeV}$ are then excluded[8] 
the statistical errors are negligible with respect to the deviations from the SM, as can be seen in fig. 2.

As we already mentioned, the differential cross-section is only very indirectly accessible to experiment, and the reconstruction of the angular distribution of $W$ 's may prove very difficult close to threshold. For that reason, experimental groups often prefer to rely on Monte-Carlo simulations to relate directly effective Lagrangian parameters to the actually observed decay products (charged leptons, jets or missing momenta). Particularly useful for this purpose is a general parametrization of the effective $W$ couplings developed by Renard and collaborators. This parametrization is in fact exhaustive for the Standard Model spectrum, with $\gamma$ and $Z$ the sole neutral bosons exchanged. As we saw above, we found it possible, and hopefully useful for future experimental analysis, to rephrase the extended model considered here (with an explicit $Z^{\prime}$ ) in terms of these parameters. We found in fact that the effect of the $Z^{\prime}$, for $e^{+} e^{-} \rightarrow W^{+} W^{-} \rightarrow$ final state, could be rephrased entirely in terms of the $\delta g, \delta \kappa$ parameters with three small caveats:

- the parametrization only applies to this channel (not to $p-\bar{p}$ production, for instance);

- the coefficients $\delta g, \delta \kappa$ become in fact form factors, depending upon $\sqrt{s}$ (which, if we neglect initial state radiation, is in practice fixed);

- we need to introduce an extra parameter $\delta g_{\gamma}$; we insist again on the fact that $\delta g_{\gamma}$ is not to be interpreted as a change in the $W$ electric charge, but only as a convenient way to simulate part of the $Z^{\prime}$ exchange.

The graphs are fairly self-explanatory, the various lines have been deliberately limited to plot only for values of $\lambda$ and $\epsilon$ leading to less than $2 \sigma$ departures in fig. 3 and show in particular that sizeable contributions to $\delta g_{Z}$ and $\delta g_{\gamma}$ are allowed, while $\delta\left(g_{Z} \kappa_{Z}\right)$ and $\delta\left(g_{\gamma} \kappa_{\gamma}\right)$ are minute. Of particular interest is the $\delta g_{Z}-\delta g_{\gamma}$ plane (fig. 4), and we think this could be used quite generally to discuss departures from the Standard Model (for instance, the present model introduces a strong correlation between $\delta g_{Z}$ and $\delta g_{\gamma}$, while non-extended cases are confined to the horizontal axis).

\section{Conclusions}

The purpose of the present paper was to investigate to what extent the severe constraints, imposed by LEP1 and the assumption of $S U(2) \times U(1)$ gauge invariance, on the possible observation of anomalous $W$ couplings at future colliders could be alleviated. We have addressed one of the deeper assumptions made in this type of approach, namely we 
have shown that a larger symmetry group could in fact lead to less constraining conditions than the minimal $S U(2) \times U(1)$. This apparent paradox stems from the fact that the linear realization of the larger group requires a larger light-mass spectrum than the minimal one usually assumed. We have only tried to produce an explicit example, involving the minimal extension of the gauge group to $S U(2) \times U(1) \times U(1)^{\prime}$, and our results do not attempt at generality (for instance, we have not included all the obviously possible extra effective terms. This example however allows us to state our point, namely that departures of the standard couplings larger than those expected for the minimal symmetry are indeed possible, provided some extra bosons are light, though weakly coupled. Such small values of the gauge couplings make the presence of $Z^{\prime}$ bosons even as light as $210 \mathrm{GeV}$ perfectly compatible with current results from hadronic colliders [9].

In the process of this analysis, we have been brought to formulate our results in terms of the Renard et al. parameters $\delta g_{Z}, \delta \kappa_{Z, \gamma}$, and we have shown that these parameters could be easily generalized to mimic in a specific channel the contribution of $Z^{\prime}$, at the cost of adding $\delta g_{\gamma}$ to their number. We have also suggested the use of correlated plots in the space $\delta g_{\gamma}, \delta g_{Z}$ to discuss the results or expectations of the various experiments. For some values of the parameters, other channels might reveal the presence of $Z^{\prime}$ earlier or even more strikingly: for instance, larger values of its direct coupling to leptons (governed by $\lambda$ ) might favour the $\mu-\bar{\mu}$ channel. This depends in each case on the set of parameters and of the experimental set-up; in any case such observability detracts in no way from the present conclusions.

\section{Acknowledgements}

The material in this paper has been the subject of numerous discussions with colleagues, either theorists or experimentalists, at CERN and during the Moriond Meeting. In particular, Alvaro De Rújula, Belén Gavela and Olivier Pène, Misha Vysotsky, Georges Gounaris, E. Massó, M. Martínez, S. Peris, Alain Blondel, Stavros Katsanevas, Daniel Treille and the DELPHI LEP200 group. 


\section{References}

[1] J-M. Frère, J.M. Moreno, J. Orloff and M. Tytgat, Phys. Lett. B292 (1992) 348.

[2] A. De Rújula, B. Gavela, P. Hernández and E. Massó, Nucl.Phys. B384 (1992) 3.

[3] M. Bilenky, J.-L. Kneur, F.M. Renard, D. Schildknecht, Bielefeld preprint, BI-TP-9225 (1992)

[4] C. De Clercq, ULB preprint IISN0379-301X (1993), to appear in "Rencontres de Moriond" proceedings (Electroweak session, march 1993)

[5] S. Weinberg, PhysicaППA96 (1979) 327; H. Georgi, Weak Interactions and Modern Particle Theory Benjamin-Cummings, Menlo Park, CA (1984).

[6] P. Hernandez and F.J. Vegas, Phys. Lett. B307 (1993) 116-127;

R. Escribano and E. Massó, Phys. Lett. B301 (1993) 419.

[7] C.L. Bilchak and J.D. Stroughair, Phys. Rev. Lett. D30(1984) 1881.

[8] F. del Aguila, W. Hollik, J.M. Moreno and M. Quirós, Nucl. Phys. B372 (1992) 3.

[9] CDF collaboration, F. Abe et al Phys. Rev. Lett. 65, 2243 (1990) 


\section{Figure Captions}

Fig. 1. Deviations from the Standard Model of low-energy observables $\left(M_{W}, e_{A}\right.$ and $\left.e_{V}\right)$ for $M_{Z^{\prime}}=210$ or $300 \mathrm{GeV} / \mathrm{c}^{2}$. Different curves correspond to different values of $\lambda$, ranging from 0 (plain curve) to 0.2 (smallest dashes), while the dependence on $\varepsilon$ can be followed along a given curve. All curves here, and in what follows, are limited to the portion where they satisfy the simultaneous constraints from LEP1 data and $\left|\delta M_{W}\right|<0.52 \mathrm{GeV} / \mathrm{c}^{2}$, i.e. inside a $2 \sigma$ departure from current values.

Fig. 2. The asymmetry $A_{135}$ as defined in (5.1)for $\sqrt{s}=200 \mathrm{GeV}, M_{Z^{\prime}}=210 \mathrm{GeV}$, as a function of $\varepsilon$ and for $\lambda$ ranging from 0 (plain curve) to 0.2 (smallest dashes). The SM would correspond to the maximum of the plain curve.

Fig. 3. The relative deviations from the SM triple-gauge couplings induced at $\sqrt{s}=$ $200 \mathrm{GeV}$ by a $Z^{\prime}$ of $210 \mathrm{GeV}$, as a function of its anomalous coupling $(\varepsilon)$, and for a gauge coupling ranging from $\lambda=0$ (plain curve) to $\lambda=0.2$ (small dots). Figures for other values of $\sqrt{s}$ and $M_{Z^{\prime}}$ can be obtained to a very good accuracy by a simple rescaling according to the dependence manifest in (4.5), (4.6).

Fig. 4. The deviations $\delta g^{Z} / g_{Z}$ (horizontally) versus $\delta g^{\gamma} / g_{\gamma}$ (vertically). As previously, a curve corresponds to a continuous change in the value of $\varepsilon$, while increasingly dotted lines correspond to $\lambda$ increasing from 0 to 0.2 . 


\begin{tabular}{|c|c|c|c|c|c|c|c|c|}
\hline Hypercharge & $\left(\begin{array}{c}u \\
d\end{array}\right)_{L}$ & $u_{L}^{c}$ & $d_{L}^{c}$ & $\left(\begin{array}{c}\nu \\
e\end{array}\right)_{L}$ & $e_{L}^{c}$ & $\nu_{L}^{c}$ & $\left(\begin{array}{l}\phi_{1} \\
\phi_{2}\end{array}\right)$ & $\chi$ \\
\hline$Y$ & $\frac{1}{6}$ & $-\frac{2}{3}$ & $\frac{1}{3}$ & $-\frac{1}{2}$ & 1 & 0 & $\frac{1}{2}$ & 0 \\
$Y^{\prime}$ & $\frac{1}{5}$ & $\frac{1}{5}$ & $-\frac{3}{5}$ & $-\frac{3}{5}$ & $\frac{1}{5}$ & 1 & $-\frac{2}{5}$ & 1 \\
\hline
\end{tabular}

Table 1: Matter content and charges assignment of the extended model $S U(2) \times U(1) \times$ $U(1)^{\prime}$.

Fig. 1: Deviations from the Standard Model of low-energy observables $\left(M_{W}, e_{A}\right.$ and $\left.e_{V}\right)$ for $M_{Z^{\prime}}=210$ or $300 \mathrm{GeV} / \mathrm{c}^{2}$. Different curves correspond to different values of $\lambda$, ranging from 0 (plain curve) to 0.2 (smallest dashes), while the dependence on $\varepsilon$ can be followed along a given curve. All curves here, and in what follows, are limited to the portion where they satisfy the simultaneous constraints from LEP1 data and $\left|\delta M_{W}\right|<0.52 \mathrm{GeV} / \mathrm{c}^{2}$, i.e. inside a $2 \sigma$ departure from current values.

Fig. 2: The asymmetry $A_{135}$ as defined in (5.1)for $\sqrt{s}=200 \mathrm{GeV}, M_{Z^{\prime}}=210 \mathrm{GeV}$, as a function of $\varepsilon$ and for $\lambda$ ranging from 0 (plain curve) to 0.2 (smallest dashes). The SM would correspond to the maximum of the plain curve.

Fig. 3: The relative deviations from the SM triple-gauge couplings induced at $\sqrt{s}=$ $200 \mathrm{GeV}$ by a $Z^{\prime}$ of $210 \mathrm{GeV}$, as a function of its anomalous coupling $(\varepsilon)$, and for a gauge coupling ranging from $\lambda=0$ (plain curve) to $\lambda=0.2$ (small dots). Figures for other values of $\sqrt{s}$ and $M_{Z^{\prime}}$ can be obtained to a very good accuracy by a simple rescaling according to the dependence manifest in (4.5), (4.6).

Fig. 4: The deviations $\delta g^{Z} / g_{Z}$ (horizontally) versus $\delta g^{\gamma} / g_{\gamma}$ (vertically). As previously, a curve corresponds to a continuous change in the value of $\varepsilon$, while increasingly dotted lines correspond to $\lambda$ increasing from 0 to 0.2 . 